\title{
HOUSE INTERACTIVE MANAGEMENT SYSTEM
}

\author{
Ishan Bisht \\ BCA Industry Oriented Program \\ of Computer Science and \\ Engineering(SCSE) \\ Uttarakhand, India
}

\author{
Jatin Soni \\ BCA Industry Oriented Program \\ of Computer Science and \\ Engineering(SCSE) \\ Rajasthan, India
}

\author{
Tushar Tyagi \\ BCA of Computer Science and \\ Engineering(SCSE) \\ Delhi, India
}

\begin{abstract}
This project is created to provide jobs to unemployed people around city or small town so that they can earn for their living. For implementing this project, we are using Microsoft Visual Studio (2019) for the front-end and SQL Server-Database for the back end. By this project you can insert your details and register yourself as a customer. After registration, you can select type of worker to get your work done. This application is created to increase work flow and increase the jobs for people.
\end{abstract}

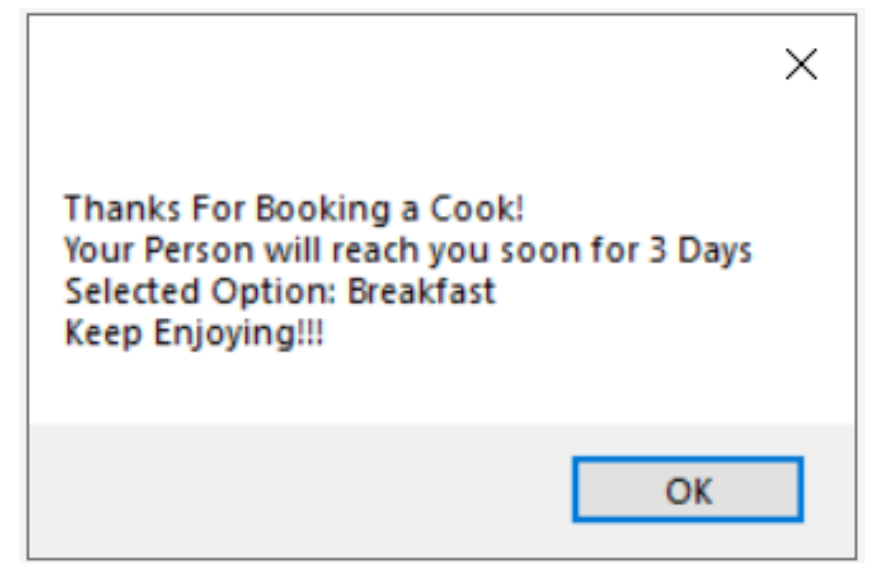

\section{INTRODUCTION}

HIMS is a desktop application which will help you to hire experts. It is an application by which one can hire a professional worker who will help you in almost every task which they can perform. It helps you to get your work done in a very short time and with cheap amount of payment.

This application works under a small area ranging from a town to a city. The performers will earn according to the work which they had done in their time.

The application starts with the login page which will lead you to the main dashboard where you can find various selection of categories to get your work get completed in a shorted duration. The main dashboard will lead you to go in your own specified direction by simply clicking on a single button.

\section{MODULES}

- Maintaining the Integrity of the Specifications

The performer is strictly taught to what he is going to perform during his task. The performer is bound to some company's policy about the behaviour with their seeker. proper punctuality and maintenance. They will be live on YouTube page so that Seekers can keep track of there live work completion and so that you wont need to worry about them and this will ultimately lead you to gain more trust to our system software in a better and effective manner.

- Problem Formulation

The problem from this project which can arise can be of self-security. In the case of workers coming home like plumber, technician, car wash man to take your car, there will be the risk of robbery, attacks. To avoid such happenings, workers must be strictly told to have a work-towork relationship with the customers in order to provide better service and consistency between organization and customers.

The worker can be identified with their outfits according to their respective jobs which they perform so those can be trusted ones and reliable also. Public will get their work done with cheapest rates available so there will be no bargaining between organization and the customers and the workers. Public will then doesn't require to have a burden of completing by going.

- Objectives

This Application will Increase Work Flow Rapidly in no time.

- Increase loyalty among everyone.

- Carefree mind.

- Providing Clean Services.

- Increased Security to Households.

- Completion of tasks in Optimum Resources.

- Saves Time and Energy. 
- Consequences Reduction.

- No Bargaining, just Performing.

- $\quad$ Literature Review

This project required information of work from each field. Relevance of this project is strong because of unemployment.

Authority of the project is hold by its creators.

In terms of Accuracy, this project will become boon to the area's economy where it is implemented.

The main purpose of this project is to decrease poverty, unemployment, scarcity for work.

Reliability of person will be kept under good considerations. Responsibility of the work done by our member will be his own complete responsibly.

- Plan

Complete work will be measured during the work, before the work, after the work.

Statistical report will be maintained during work and weekly status will be measured in order to attain the most accurate report.

Graphs will be used for the data representation.

Proper methodology will be used.

Proper background inspection will be there.

Proper measures will be taken while controlling the sequence of the ongoing task in a better way.

- Analysis

Financially this project will be cheaper whereas in productivity, it will be at top charts.

According to time, this project will be better performer since it will be used thoroughly.

Technically it will be supporting every smart phone (both iOS and Android), along with tablets. (currently under development).

Strategy says that the market will grow unexpectedly because of customer needs, demand, and no competition.

The strategies which we are using are according to the laws, regulations, and standards.

This project will incur the organizational change in culture of the people living around.

Build trust among each other, which will automatically lead one to the direction of Peaceful Mindset.

\section{STEPS FOR CREATING C\# WINDOWS FORM}

1. Open Visual Studio 2019.

2. Choose Create a new project.

3. Choose the Windows Forms App (.NET Framework) template.

4. Type 'name of project' in the Project name box.

5. Choose Create.

\section{STEPS FOR ADDING CONTROLS}

1) Choose Toolbox to open the Toolbox fly-out window. View $>$ Toolbox. Or, press Ctrl + Alt + $\mathbf{X}$.

2) Choose any type of control and then drag it onto the form.

\section{STEPS FOR ADDING CODE INTO FORM}

- Drag control(button) from toolbox into form.

- Double click on control to add code into cs formatted file which will work on $\mathrm{C \#}$ language.

\section{FOR RUNNING FORM APPLICATION,} press the green play button.

\section{Software Requirements:}

They are the specifications which are needed to run a particular application or software.

\begin{tabular}{|l|l|}
\hline Number & Description \\
\hline 1 & Windows 7,8,10 \\
\hline 2 & C\#(.NET Framework) SQL Server Database \\
\hline 3 & SQL Server Database \\
\hline 5 & IDE: Visual Studio 2019 \\
\hline 6 & C\# Windows Form Application and SQL Queries \\
\hline
\end{tabular}

\section{Hardware Requirements:}

They are the Physical components of a system which are required to run a particular application or software.

\begin{tabular}{|l|l|}
\hline Item & Description \\
\hline CPU & Windows 7,8,10 \\
\hline RAM & C\#(.NET Framework) SQL Server Database \\
\hline PROCESSOR & SQL Server Database \\
\hline
\end{tabular}

There lies a difference between both which is that the software focuses on inner modules to be covered in order to meet a certain condition, whereas, hardware focuses on body or physical components in order to meet the minimum requirements.

System Design

1. Activity Diagram

2. Data Flow Diagram

a. Level Zero

b. Level One

3. Entity Relationship Diagram

4. Use Case Diagram 
Activity Diagram

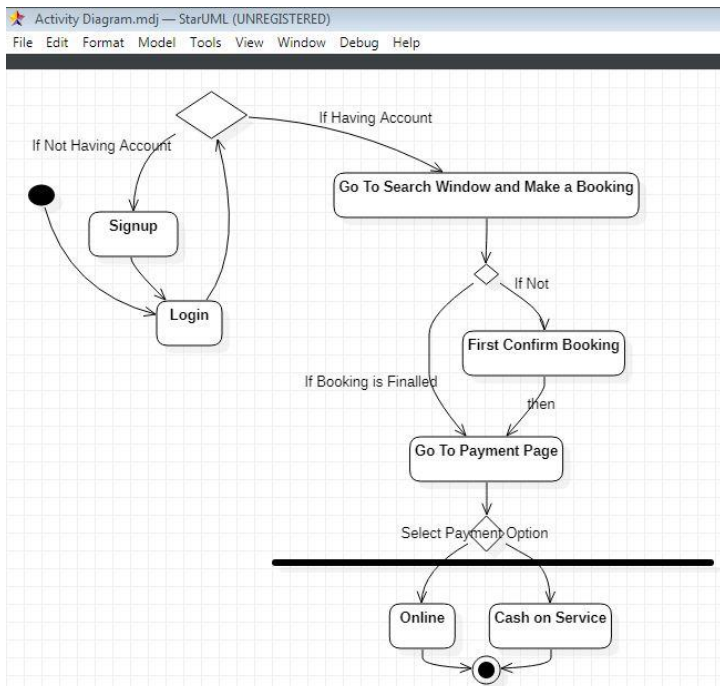

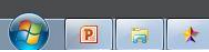

DataFlow Diagram

1. Level Zero

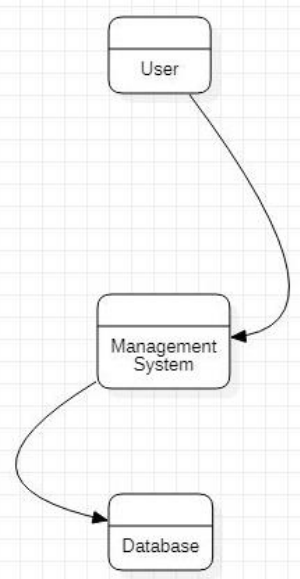

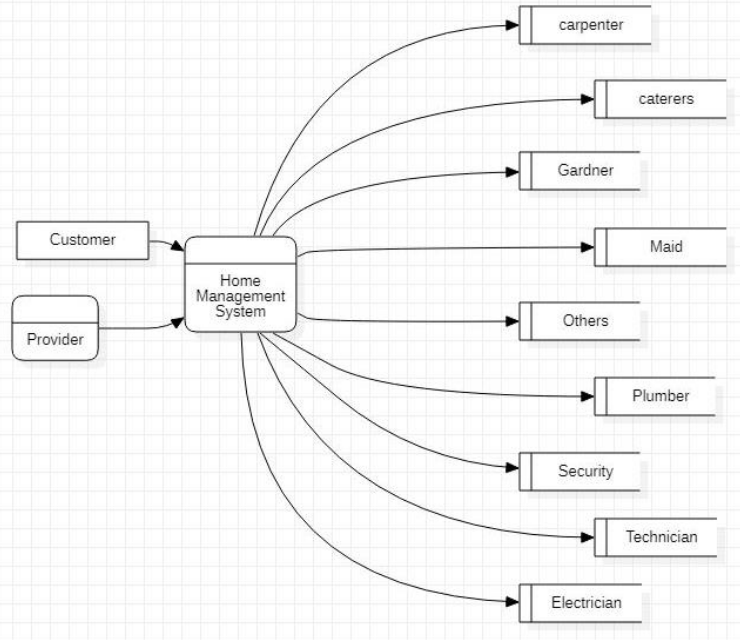

ER Diagram

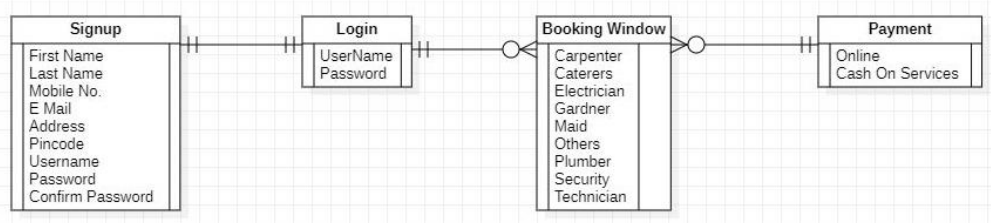

Use Case Diagram

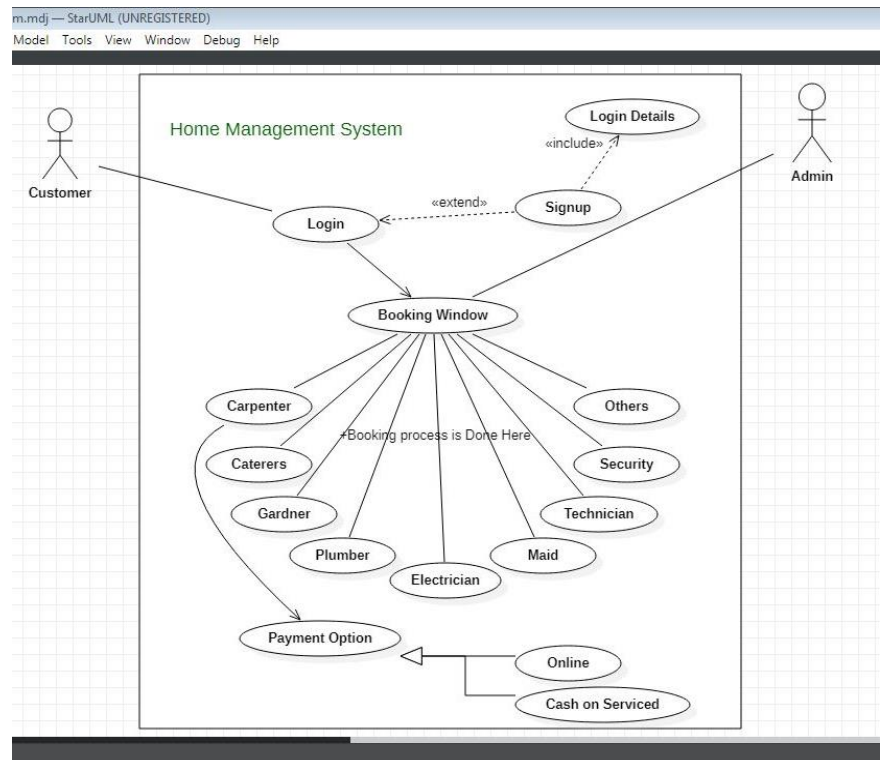

Q t

2. Level One 
Published Online March 2021 in IJEAST (http://www.ijeast.com)

Screenshots of Project

1. Login Page
3. Main Page / Dashboard

\section{+IIMS}

\section{+IIMS}

\section{S Username: Jitu \\ Password: $\bullet \bullet \bullet$}

\section{LOGIN}

Don't Have an Account? Signup»

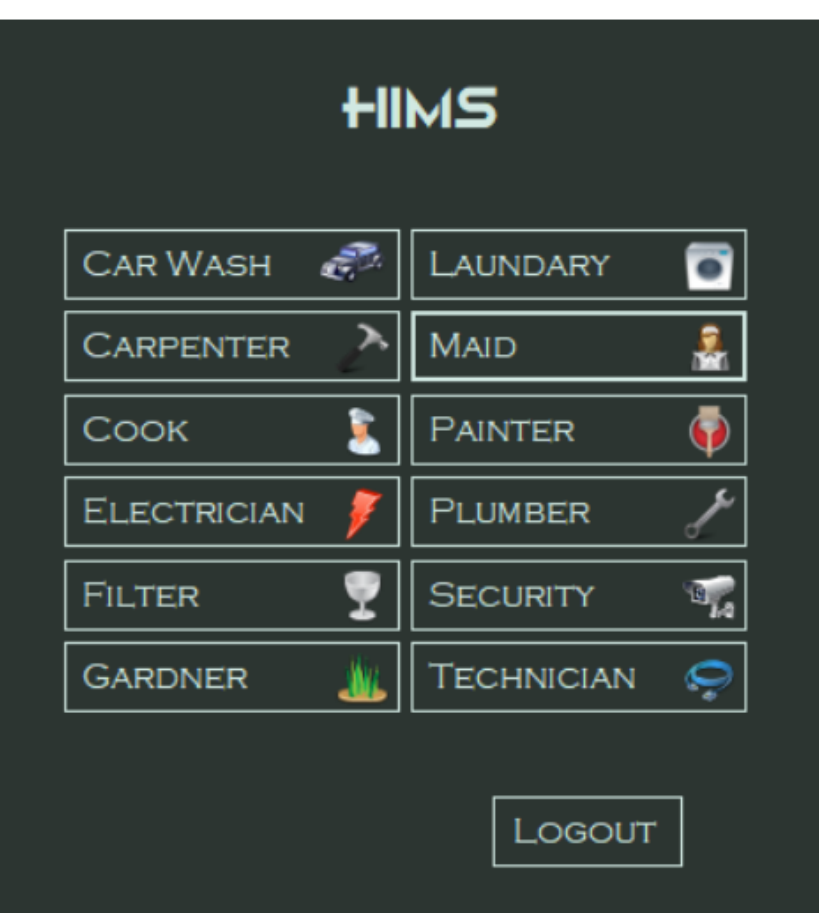

4. Car Wash

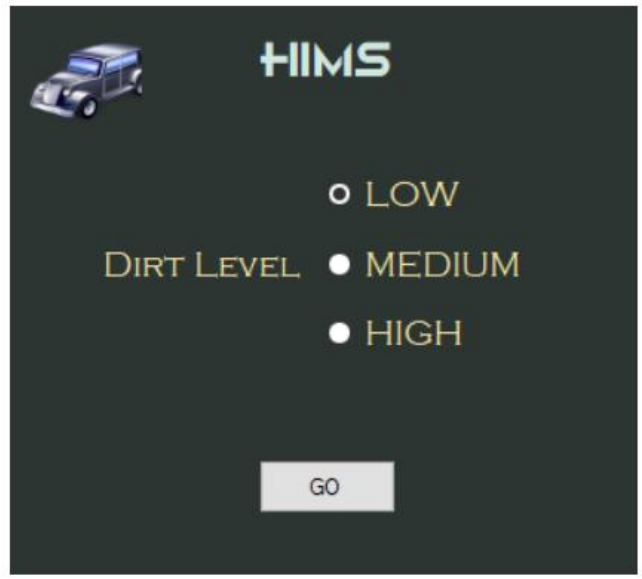

Already have an Account? Login» 
International Journal of Engineering Applied Sciences and Technology, 2021

Vol. 5, Issue 11, ISSN No. 2455-2143, Pages 304-313

Published Online March 2021 in IJEAST (http://www.ijeast.com)

5. Carpenter

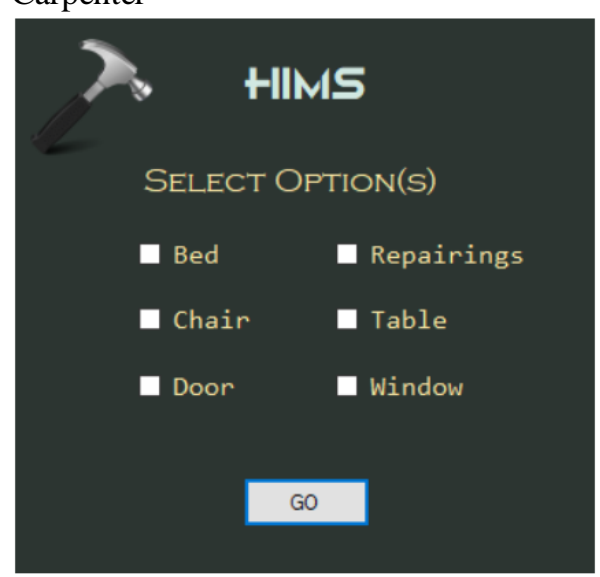

6. Cook

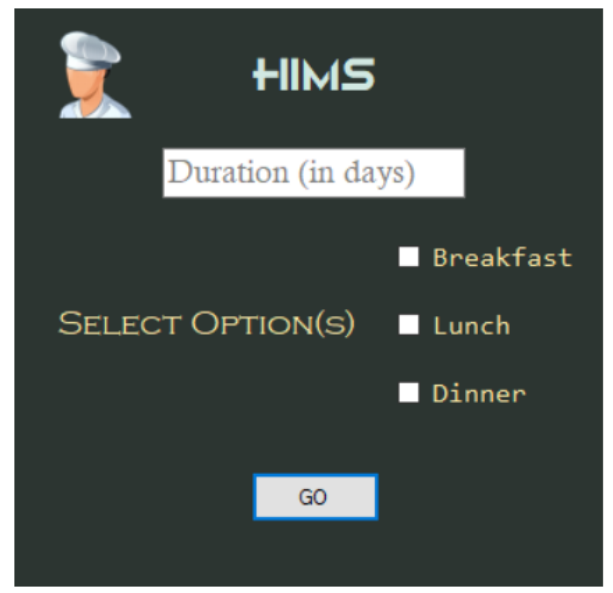

7. Electrician

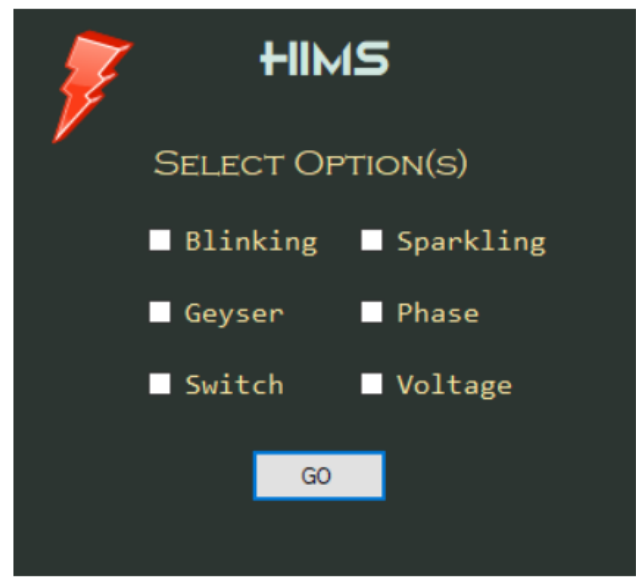

8. Filter

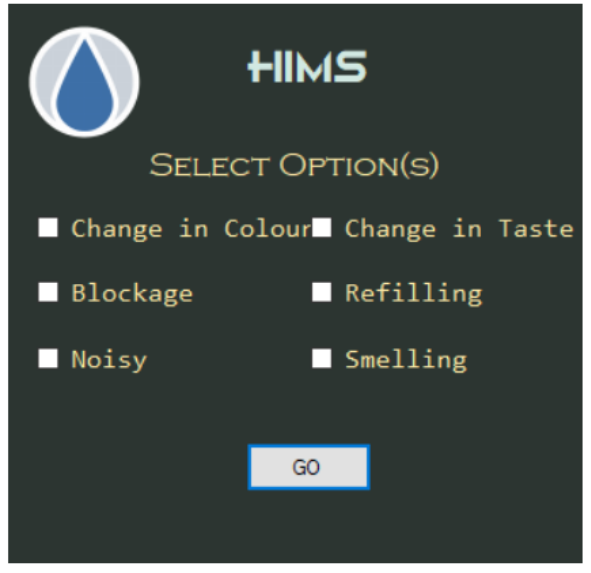

9. Gardner

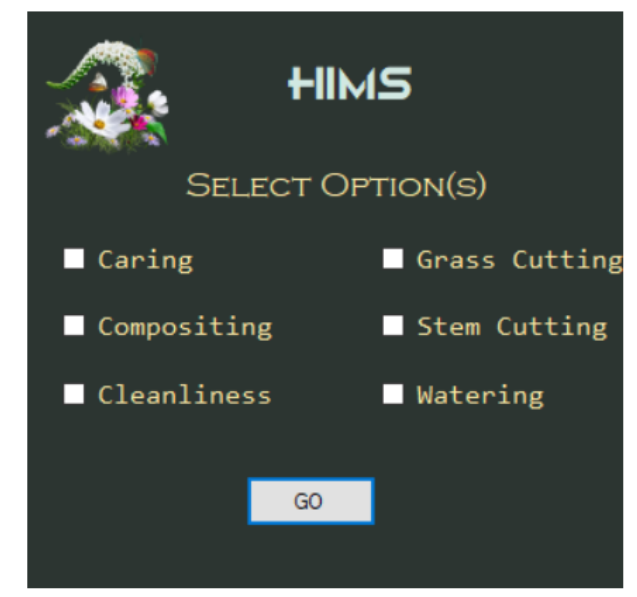

10. Laundary

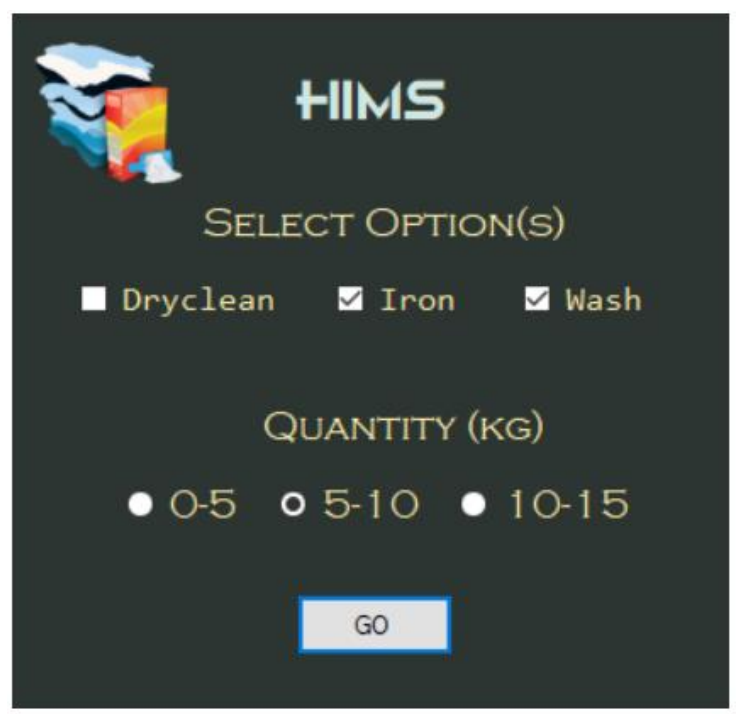

11. Maid 
International Journal of Engineering Applied Sciences and Technology, 2021

Vol. 5, Issue 11, ISSN No. 2455-2143, Pages 304-313

Published Online March 2021 in IJEAST (http://www.ijeast.com)

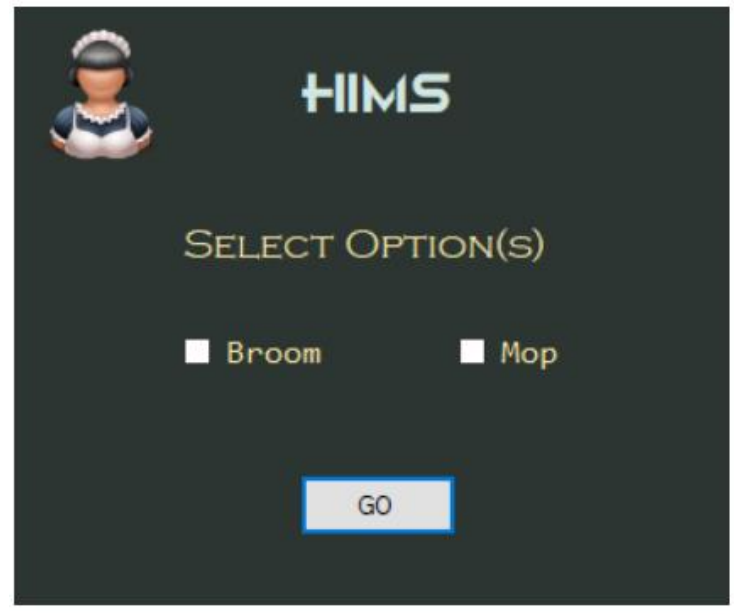

12. Painter

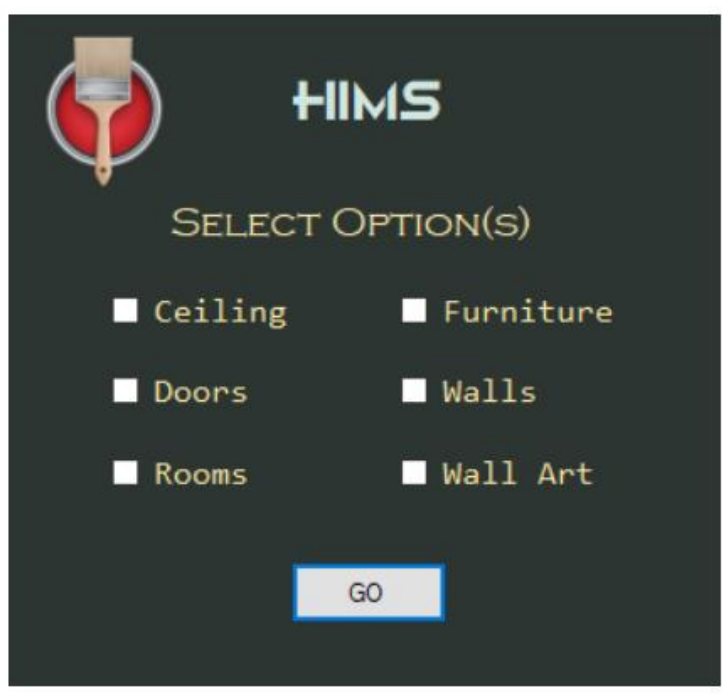

13. Plumber

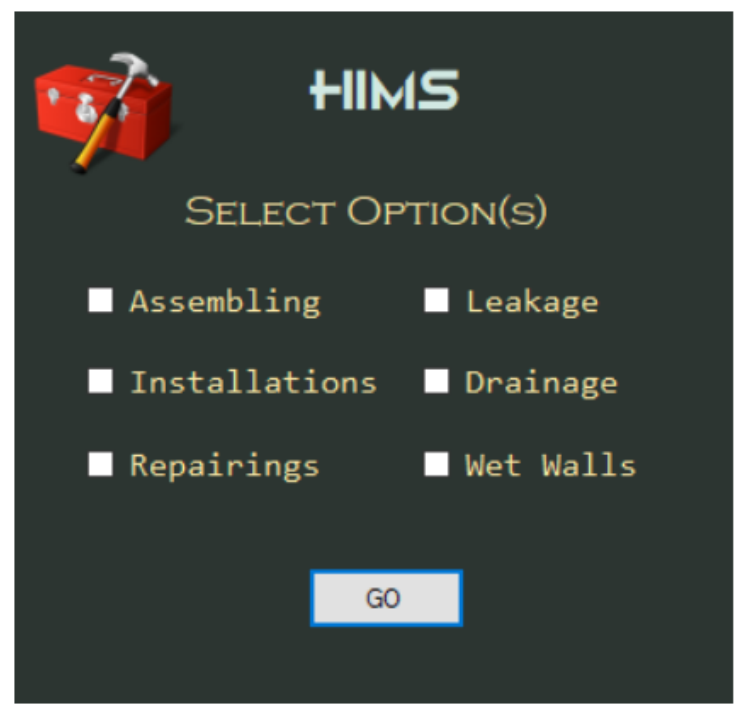

14. Security

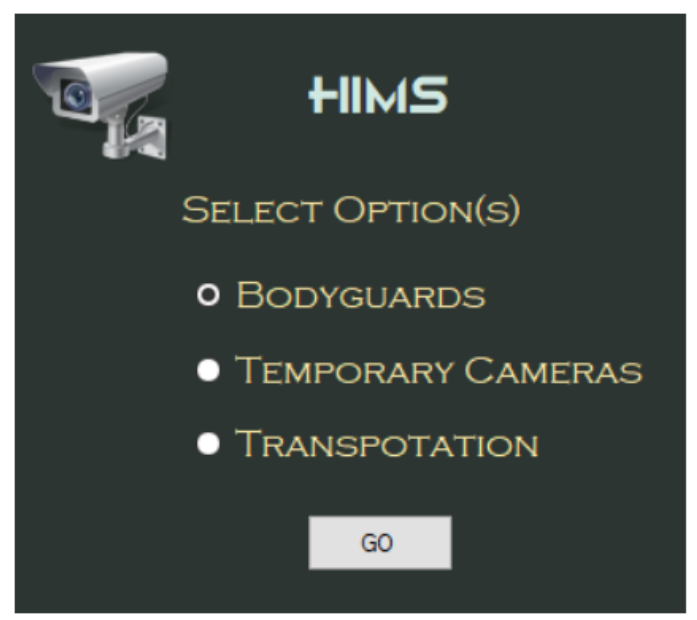

15. Technician

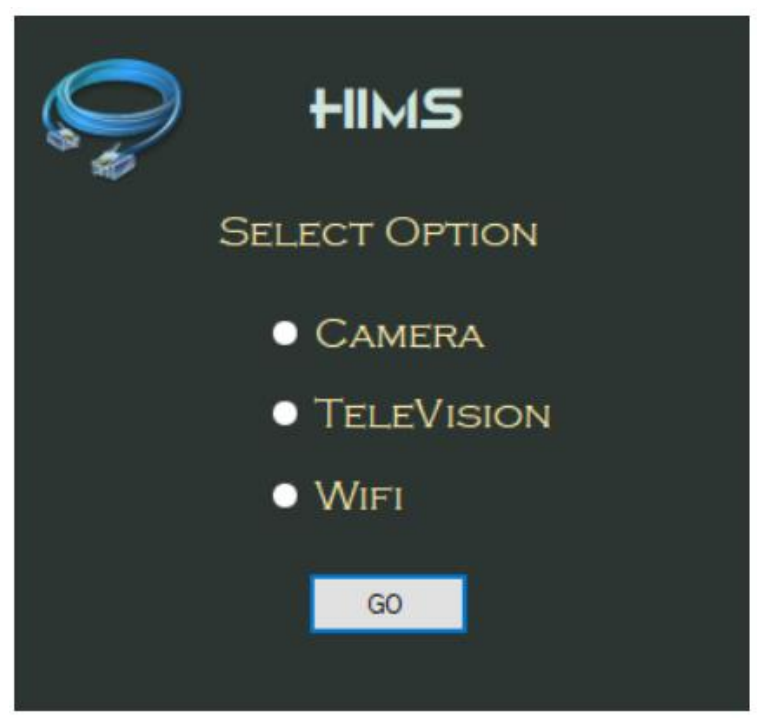

Database Table:

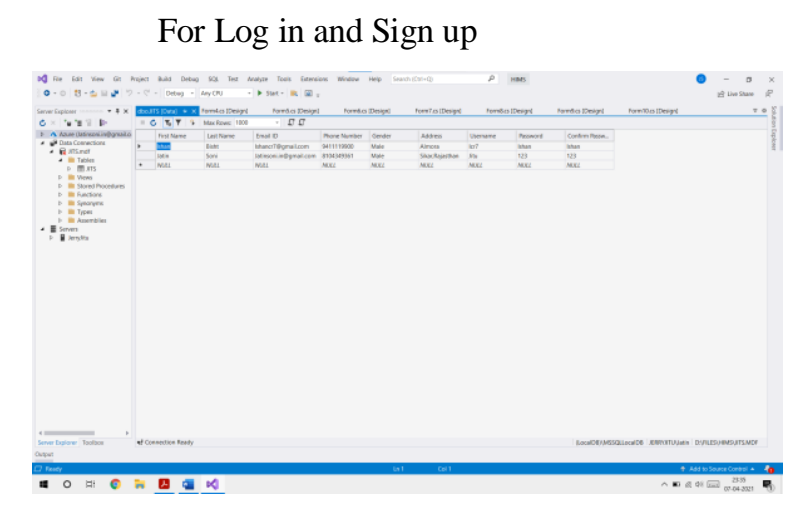

IV. CONCLUSION

The Solution is completed successfully with taking the help of teachers and courses which are available over the internet in a broad variety by different organizations and also on YouTube. We are rewarding our team for supporting us up 
to this level so that we are able to complete our project today.

Goals for future:

a) Goal for $1^{\text {st }}$ Quarter:

i) Increase in Customer Satisfaction by $2 \%$

ii) Maintain Growth

iii) Employee Perks and Pre-requisites

b) Goals for $2^{\text {nd }}$ Quarter:

i) Ensuring the cost of development stays below budget

ii) Tournaments

iii) Food Drives

iv) More employee Opportunities

\section{REFERENCES}

1) Udemy

\section{https://www.udemy.com/topic/visual-studio/}

2) LinkedIn

https://www.linkedin.com/learning/topics/visualstudiol

3) Pluralsight

https://www.pluralsight.com/paths/visual-studio$\underline{2019 /}$

4) Visual Studio Code https://code.visualstudio.com/learn/

5) Edureka

https://www.edureka.co/blog/visual-studio-tutorial/

6) Microsoft

https://visualstudio.microsoft.com/vs/getting-

started/

7) Friends, Teachers, Family, Viewers.

8) Download Free Version from Here:

https://visualstudio.microsoft.com/thank-you-

downloading-visual-

studio/?sku=Community\&rel=16 